\title{
Chinese learners' perceptions towards teachers' language use in lexical explanations: A comparison between Chinese-only and English-only instructions
}

\author{
Lili Tian ${ }^{\mathrm{a},}$ *, Mairin Hennebry ${ }^{\mathrm{b}}$ \\ a School of Foreign Languages, Renmin University of China, 59\# Zhongguancun Street, Haidian District, Beijing 100872, PR China \\ ${ }^{\mathrm{b}}$ Division of English Language Education, Faculty of Education, University of Hong Kong, Meng Wah 658, Pok Fu Lam Road, Hong Kong
}

\section{A R T I C L E I N F O}

\section{Article history:}

Received 15 December 2015

Received in revised form 6 August 2016

Accepted 9 August 2016

Available online $\mathrm{xxx}$

Keywords:

Learners' perceptions

Preferences

Language use

Lexical explanation

\begin{abstract}
A B S T R A C T
This paper examines Chinese learners' contextualized perceptions of and preferences towards teachers' language use for explaining unknown lexical items, and compares the perceptions of 68 Chinese university students in the context of English-only versus Chinese-only lexical explanations. A questionnaire was administered to students to investigate their general language use preferences. 36 follow-up interviews were also conducted in order to examine students' perceptions in the context of more specific lexical explanations. While questionnaire responses suggested that a majority of students held positive attitude towards both types of explanations, interview data indicated more negative perceptions towards the lexical explanations they received. The perceived distinctions of learners towards the use of English and the use of Chinese as teachers' language choices for lexical explanations were uncovered with regard to the learning of lexis. The paper discusses the implications of the study for classroom practice specifically in the area of lexical explanations and situates the discussion in the broader context of the debate around L1 use in the L2 classroom.
\end{abstract}

(C) 2016 Published by Elsevier Ltd.

\section{Introduction}

In recent years the field of Second Language (L2) learning has experienced a pendulum swing from the promotion of exclusive L2 use in the classroom to, arguably, an 'anything goes' stance in terms of the use of L1. Indeed, it is difficult to find recent studies arguing that the use of L1 in the L2 classroom might not be altogether beneficial for L2 learning. In a recent paper, Macaro (2015) argued that teachers' use of L1 in today's classrooms has become characterized by the use of L1 as a shortcut to exams; namely he explained that teachers typically use the L1 as a faster means of covering all the material that needs to be covered in preparation for exams, rather than due to any specific pedagogical principle. Arguably this results in teachers engaging with L1 use practices that are based not on sound theory and research evidence for effective learning, but instead shaped by a wash-back effect of examinations. In this context there is a need for more nuanced understandings of how teachers might use L1 in a principled manner. One way of contributing to this aim is to focus attention on a specific area for which L1 might typically be used, namely lexical explanations (see Author 1, 2014), looking particularly at how teachers explain unfamiliar lexical items (see for instance, Author 1 et al., 2015) and on how learners perceive teachers' L1 use.

Teachers'L1 use inL2 classrooms has been a major focus of L2 researchers across a variety of contexts, but few studies have examined learners' perceptions towards teachers' language use (e.g. Levine, 2003). Furthermore, previous studies have for the most part gathered data on general perceptions not contextualized in specific practices, allowing only general implications to be drawn. Given the acknowledged significance of context in shaping perceptions and attitudes, studies focusing on learners' perceptions of teachers' specific lexical explanation behavior have the potential to yield a more fine-grained understanding of the

\footnotetext{
* Corresponding author.

Email addresses: lili.tian@ruc.edu.cn (L. Tian); mhennebr@hku.hk (M. Hennebry)
} 
way in which students react to teachers' practices, allowing focused reflection on factors contributing to effective teaching and learning of lexis.

This paper, part of a broader experimental study (Author 1 et al., 2012), focuses on Chinese learners in an EFL university context and their perceptions of teachers' use of L1, specifically in offering lexical explanations for unknown lexical items. A comparison of perceptions is drawn between instances when learners are provided with an English-only explanation and those where a Chinese-only explanation is offered. Compared with the classroom context where a mixture of both L1 and L2 lexical explanations are relatively less employed by some teachers due to limited class time (Author 1 et al., 2015), such a contextualized comparison enables researchers to elicit much clearer perceptions of learners. The reported benefits and problems of English and Chinese lexical explanations are presented and illustrated in depth.

\section{Research background}

In the 1980s, the exclusive use of L2 and more specifically immersion language environments have been deemed facilitative for language acquisition (Krashen, 1985). However, as studies on L1 use and codeswitching (CS) in L2 classrooms proliferate, the role of L1 has been increasingly advocated, with L1 seen to serve as a scaffolding strategy in constructing collaborative dialogue (Anton \& DiCamilla, 1998), as mediation between an L2 word and its concept (Kroll \& Stewart, 1994), as a compensatory strategy for lexical gaps (Ong \& Zhang, 2010; Zhang, 2002), as a tool for offering lexical and grammatical explanations (Levine, 2003), and as a medium through which to build rapport (Gao \& Dai, 2007).

Initial results from studies probing into the effects of L1 use on L2 learners' learning outcomes suggest mixed results. Some studies indicate at least some short-term acquisitional benefit to be gained from L1 rather than L2 lexical explanations (e.g. Author 2 et al., 2013; Lee \& Macaro, 2013). However, in an experimental study of 65 adult Chinese learners of English, Zhang (2002) found that Chinese L1 students who used mother tongue morphological knowledge in the retention and retrieval of English lexicon experienced less success than those who used target language morphological knowledge. Thus, further research is needed to unpack and explain the complex nature of interactions between the L1 and the L2 in the process of language learning. A key aspect of this interaction is the learners' perceptions of their teachers' lexical explanations and specifically the teachers' code choice in providing the explanations. Where research has placed an emphasis on describing the amount and functions ofL1 use, it needs also to specifically explore the perspective of the learner, as a key player in the teaching and learning process, on their teachers' specific code choice.

Previous studies have explored learners' general perceptions towards teachers' language use. In a questionnaire study conducted within a multilingual classroom, Duff \& Polio (1990) found that the majority of American university students held positive attitudes towards their teachers' language use proportions, despite the fact that theteacher'sL1 use ranged from 0 to $90 \%$. In addition, in every class, over $70 \%$ of the students claimed to understand "most" or "all" of the teacher's L2 use. Arguably, these results might be interpreted to indicate that the amount of L1 use may not be the deciding factor for students, rather the effectiveness of L1 use in allowing students to understand and keep up with the content of the lesson is of greater significance. Indeed this is supported by Macaro's (1997) large-scale study on language use in the UK classroom context. The study examined learners' perspectives on their teachers' L1 use through questionnaire and focus group interviews. The majority of participants in this study reported needing their teachers to speak the L1 sometimes in order to facilitate understanding, with many adding that they could not learn if they could not understand their teacher. Levine (2003), however, found that students who reported higher TL use in their FL classes actually tended to report lower levels of anxiety about TL use, presumably because the exposure allowed the L2 to become a familiar phenomenon to the students, thus lowering the potential sense of inhibition learners often experience when using the L2. Such findings support calls for a deeper understanding of what might constitute principled use of L1 that facilitates understanding on the one hand but provides sufficient L2 exposure on the other.

In Chinese contexts, the following studies examined the general perceptions of Chinese university undergraduates towards teachers' L1 use. Kong \& Zhang (2005), for example, found that of the $20 \%-35 \%$ of talk in the class that was teacher talk, $2 \%-5 \%$ was in the L1 (Chinese); a proportion with which over $70 \%$ of students expressed satisfaction. Examining the L1 use of four teachers, Zhong (2007) found greater variability in the amount of English used, ranging between $57 \%$ and $87 \%$.Nevertheless, $88 \%$ of the students were satisfied with the proportion of teacher's current English/Chinese use, with students citing reasons such as L1 helping establish their confidence, making them feel relaxed, encouraging them to greater participation in classroom activities and generally supporting their L2 learning. Given the fact that in both studies, students expressed satisfaction with their teachers' use of L1 despite the considerable differences, there is reason to hypothesize that what is more significant to students is the effectiveness and function of L1 use, rather than the amount. Through stimulated recall interviews, Guo (2007) investigated students' perceptions of their teachers' L1 use. 12 of the16 Chinese non-English-major undergraduates interviewed reported positive attitudes towards the use of L1 in the English classroom, explaining that for them L2 exclusivity destroyed their confidence, making them feel deprived and helpless and leaving them craving for meaning and clarity. However, the remaining four students expressed negative opinions towards teachers' L1 use. While these students were in favor of L1 use for the purpose of explaining low frequency vocabulary, they found it difficult to see the purpose of L1 use for cases where students could easily understand 
the L2, ${ }^{1}$ calling into question the pedagogical principles underpinning such instances. As with Kong \& Zhang (2005) and Kong (2007), Guo's study again points towards the importance of understanding the pedagogical function and effectiveness of L1 use, thus necessitating a focus on specific instances.

Studies that have focused on specific instances of L1 use, rather than on general impressions, are few. Through a large-scale study involving questionnaires to 798 adult and young students and interviews with 22 students, Macaro \& Lee (2013) explored Korean students' attitudes towards English-only instructions versus instructions containing some switching to L1 for lexical explanations. While adult learners expressed significantly more preference for teachers' English-only lexical explanations than young learners of age 12 if the English explanations were understandable, both age groups preferred a bilingual approach to vocabulary explanations. However, it should be noted that, the given statement in the questionnaire to which participants were asked to respond, specifically elicited perceptions of a bilingual approach ("I prefer my teacher to use English and Korean in teaching English vocabulary because using both languages will result in better understanding”). It is perhaps unsurprising that this was the preferred mode of instruction for all age groups when responding to such a given statement. Indeed, in many cases it is a bilingual approach that is adopted in the language classroom. Author 1 et al. (2015), for instance, investigated three university Chinese teachers' lexical explanations. A mixture of L1 and L2 explanations was used by all the three teachers, but at different amounts, ranging from $7.7 \%$ to $50 \%$ of total lexical explanations. Once again, however, such research points towards the quantity of L1 usage, but tells us little about students' perceptions of the usage in the specific instances, with regard to learning of lexis. It is clear that more work is needed to elicit learners' contextualized perceptions on language use by a sharp comparison between an English-only versus a Chinese-only instruction mode for lexical explanations, to allow an understanding of the distinctions between the two different linguistic mediums and the ways in which each interacts with learning.

Although existing studies have tended to conclude that the majority of learners are satisfied with their teachers' L1 use, studies on learners' general perceptions towards teachers' L1 use have typically not distinguished between different functions, thus providing general, rather than specific, insights for teachers' L1 practice. Building on previous research, there is now a need to develop more specific pedagogical implications, through close examination of learners' perceptions, rooted in teaching practices in the context of L1 use in the L2 classroom.

In light of this, the present study sought to answer the following research questions:

1) What are students' perceptions of teachers' Chinese-only and English-only lexical explanations in English language classes?

2) What are students' preferences of teachers' Chinese-only and English-only lexical explanations in English language classes?

3) In what ways do Chinese students believe English-only and Chinese-only lexical instructions interacting with their learning?

\section{The research design}

The study reported here constitutes part of a larger research project investigating the effects of teachers' L1 use on Chinese learners' vocabulary learning. A quasi-experimental method was employed to investigate the varying effects of teachers' different language use, in explaining target vocabulary in the context of listening comprehension. After the six-week intervention, interviews and questionnaires were administered to gather students' contextualized perceptions towards the linguistic medium through which they received the teacher's lexical explanations. The study thus offered an immediate context in which learners could ground their perceptions, so as to allow them to develop clear and specific beliefs on the different linguistic mediums of lexical explanations. A detailed account of the parent study is available in Author 1et al. (2012). The following is a description of the methods and procedures implemented for gathering data on student perceptions.

\subsection{Sample}

117 first-year university students, majoring in English from one university in Shandong Province of China participated in the survey. Perceptions and preferences of 68 out of the 80 students $^{2}$ from the two experimental groups are compared in the context of English-only versus Chinese-only explanations. The 37 students in the control group, who received no lexical explanations, also expressed their preferences towards teachers' language use.

Student participants were aged around 19 years old and had completed high school education with eight years' English study. They were at a low-intermediate level of English proficiency. As English major students, they were required to engage with 20 h' compulsory and intensive English instruction per week.

All participants were randomly allocated to three groups; two experimental groups (L1 or L2 lexical explanations) and one control group (no lexical explanations) (see Fig. 1).

\footnotetext{
${ }^{1}$ E.g. The teacher said ' 1994 ' in Chinese, which some students felt they could easily have understood had the teacher said it in English.

2 The questionnaire and interview were conducted after the delayed vocabulary posttest rather than after each immediate posttest, so after six-week intervention there were drop-outs. 102 valid questionnaires were received, including 68 from the two experimental groups and 34 from the control group. 36 students attended the interview afterwards.
} 


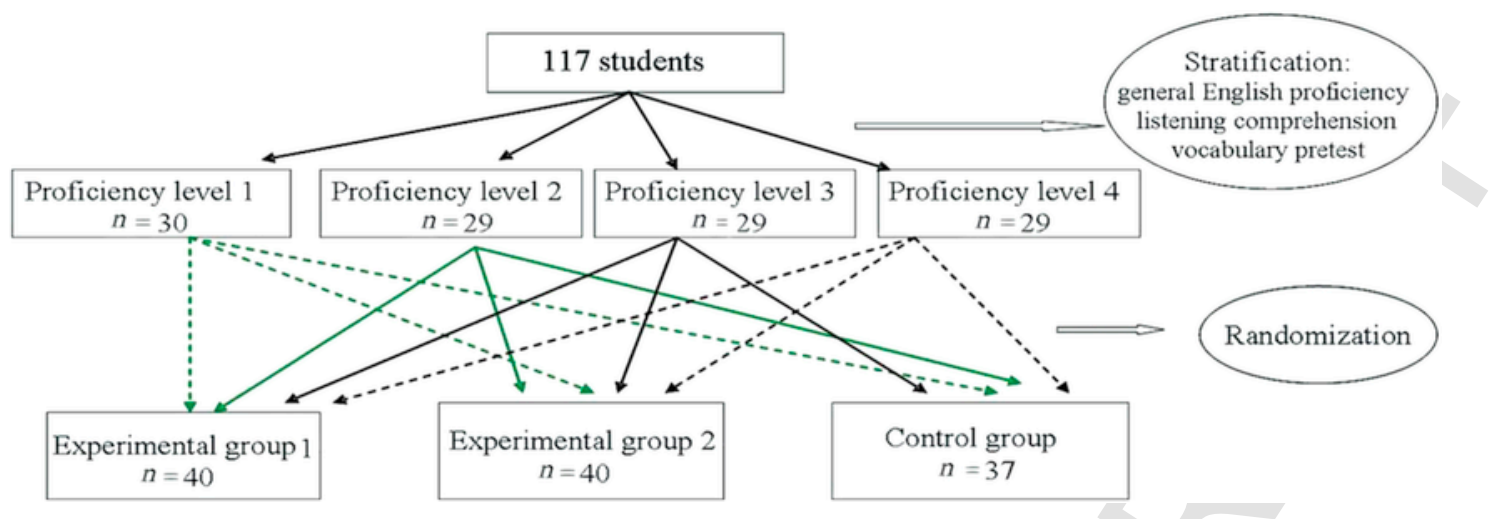

Fig. 1. Stratified random allocation to the three conditions (taken from Author 1 et al., 2012:374).

\subsection{Procedures}

Following the six-week intervention referred to above, students were invited to complete a questionnaire examining their perceptions of the teachers' use of language for lexical explanations during the intervention; 102 valid questionnaires were received. About a third of the students from each of the groups were randomly selected for interview; 36 students, evenly distributed across the three groups, accepted the invitation. This number of interview participants was deemed the maximum manageable amount for eliciting quality interview data.

The questionnaire and interview were conducted after the delayed vocabulary posttest rather than after each immediate vocabulary posttest, ${ }^{3}$ in order to avoid over-surveying the participants. Since there were six immediate posttests, following each one with a questionnaire and interview would have resulted in participant fatigue, potentially invalidating the responses.

\subsection{Instruments}

The design of the questionnaire (see Appendix) was based on a tool developed by Levine (2003), and was modified based on results of piloting. This paper focuses on data generated on the comparison between the two experimental groups' perceptions of teachers' actual practices as they directly experienced them. The questionnaire for the control group who did not receive teacher's lexical explanations was tailored accordingly. In other words, control group participants were asked only about their general preferences of language use for teachers' lexical explanation.

The questionnaire for the experimental groups targeted two areas: learners' perceptions of the experimental teacher's language use for the specific function of lexical explanation in the context of the intervention; learners' preferences of teachers' language use for lexical explanation. These areas for investigation were selected for the following reasons. Firstly, learners' perceptions of the teacher's language use during the experiment narrows down their perception and focuses on one of the most common purposes of L1 use, teacher's lexical explanation. This allowed for greater specificity on the underlying causes for their perceptions, thus contributing to our understanding of the impact of teachers' lexical explanation behavior on learners' learning. Secondly, exploring learners' preferences of teachers' language use for lexical explanation gives further insights into what students believe facilitates or hinders their language learning, helping to inform implications for teachers' language choices during lexical explanation behavior.

Semi-structured interviews with individual students (see Appendix) helped further elicit a more fine-grained understanding of the reasons for learners' views and particularly allowed for follow-up on points of interest emerging from questionnaire responses. Interview questions were tailored to reflect the variation in the training that the different groups received. Based on previous literature (e.g. Guo, 2007), the issues addressed in the interviews were: whether lexical explanations from the teacher were helpful in terms of both comprehension and memorization of target words; students' preference of teachers' language choice towards vocabulary explanation and the reasons for such preferences. The interviews were conducted in Chinese, in three different rooms for three different groups by three different researchers. One of the interviewers is one of the authors of this paper, while the other two were experienced teachers working in the sample university. The two teachers were trained beforehand on the purposes of the research, the questions to ask students and the procedures for the interviews. Mock interviews were conducted in order to provide practice for the teacher-interviewers and to allow for a degree of standardization across the interviews. Each inter-

\footnotetext{
${ }^{3}$ These vocabulary tests were reported in the parent study in details (Author 1 et al., 2012), aiming to measure the effects of teachers' L1 use on the participants' vocabulary learning outcome.
} 
view lasted for about $20 \mathrm{~min}$. All the interviews were audio-taped, giving the researcher an opportunity to verify the comparability of interviews across the interviewers.

\subsection{Data analysis}

Analysis of learners' perceptions of the teacher's language use for specific lexical explanations draws on both the quantitative and qualitative data from the questionnaire. All questionnaire data was keyed into Excel for storage and SPSS for statistical analysis.

Interview data was first transcribed verbatim, then analyzed qualitatively with a focus on providing answers to the research questions. Broadly speaking the analysis followed the stages proposed by Miles \& Huberman (1994) for generating meaning from qualitative data. The stages involve counting frequencies of occurrences, noting patterns and themes, using informed intuition to examine plausibility, clustering data into categories, types and classifications, connecting data with theory factoring (bringing a large number of variables under a smaller number of frequently observed hypothetical variables), building a logical chain of evidence drawing inferences, hypothesizing relationships between variables and finally developing theories to explain the phenomena. A constant comparison approach was then adopted, allowing the researchers to move iteratively between the themes with a specific focus on the motives supporting the attitudes and perceptions of the student participants.

\section{Findings and discussions}

Quantitative data is presented first to provide a general landscape of student perceptions and preferences of lexical explanations so as to address the first and second research questions. In answer to our third research question, qualitative data is then presented and discussed to reveal in detail the different ways Chinese translations and English lexical explanations were perceived by students interacting with learning.

\subsection{Contextualized perceptions towards teachers' lexical explanations}

It can be seen from Table 1 that, 97.1\% of students from the English-only group were satisfied either most or all of the time, while $81.8 \%$ of students from the Chinese-only group were satisfied. A non-parametric independent samples T-test on group differences revealed no significant difference in levels of satisfaction between the two experimental groups. The majority of students in both groups expressed satisfaction with the lexical explanations regardless of the linguistic medium. This finding is consistent with that of Duff \& Polio (1990) where the majority of students were satisfied with the current amount of L1 used by their teachers regardless of what that amount actually was (from 0 to $90 \%$ ). The finding further echoes that of Kong \& Zhang (2005) who found that over $70 \%$ of students were satisfied with teacher's L1 use (2-5\%).

Similarly, when asked whether students believed their teacher's lexical translations helpful, a majority from both experimental groups expressed positive views. $80 \%$ of students from the English-only group felt the teachers' use of L2 for lexical explanation was helpful most or all of the time, while $66.7 \%$ of students from the Chinese-only group felt the same about their teachers' use of L1 for lexical explanations. Although the proportion of positive responses was less among the Chinese-only group than the English-only group, the difference was not significant. This suggests that, as might be expected, students' satisfaction with their teachers' lexical explanations, is underpinned by the extent to which they find the explanation helpful.

Nevertheless, close examination of participants' qualitative responses indicates less satisfaction and helpfulness than the quantitative responses. Qualitative data gathered from the interviews suggested that about half of the views expressed about teachers' lexical explanations, were in fact negative. This incongruity between the questionnaire and the interview data points towards the need for research to look more closely at students' perceptions and go beyond the general impressions gathered by questionnaires. The analysis of the interview data in this study offers a valuable and more nuanced insight into students' reactions to their teacher's language choice in the context of lexical explanations, which will be reported in the qualitative findings in depth.

\subsection{General preferences towards teachers' lexical explanations}

All three groups were asked to express their general preferences towards teachers' language choices (English-only; Chinese-only; a mixture of both Chinese and English) in providing lexical explanations in English language classes. Questionnaire data shows that $56.9 \%$ of all participants preferred teachers to use both Chinese and English for vocabulary explanations, while almost half of the students (43.1\%) were in favor of exclusive L2 use for lexical explanations (see Table 2). None of the participants supported the exclusive use of Chinese for this purpose.

No matter what linguistic medium the students received in the lexical explanations, in general they all either prefer English-only vocabulary explanation or a mixture of both Chinese and English. The Control Group who received no lexical explanation also reported the same tendency as the other two experimental groups. Of course at one level, we might argue that this is not a surprising finding; it would be unexpected for students to express a preference for exclusive L1 use for all lexical explanations. 
Table 1

The perceived helpfulness and satisfaction of the two experimental groups towards their teacher's lexical explanation.

\begin{tabular}{|c|c|c|c|c|c|c|c|c|c|c|}
\hline & & $1=$ not at all & $2=$ most of the time not & $3=$ half of the time & $4=$ most of the time & $5=$ all the time & $\mathrm{N}$ & Mean & $\mathrm{SD}$ & Sig. \\
\hline \multirow[t]{2}{*}{ Satisfaction } & English-only group & 0 & 0 & $2.9 \%$ & $44.2 \%$ & $52.9 \%$ & 35 & 4.5 & 0.564 & $>0.05$ \\
\hline & Chinese-only group & 0 & 0 & $18.2 \%$ & $24.2 \%$ & $57.6 \%$ & 33 & 4.39 & 0.788 & \\
\hline \multirow[t]{2}{*}{ Helpfulness } & English-only group & 0 & $5.7 \%$ & $14.3 \%$ & $51.4 \%$ & $28.6 \%$ & 35 & 4.03 & 0.822 & $>0.05$ \\
\hline & Chinese-only group & 0 & $12.1 \%$ & $21.2 \%$ & $27.3 \%$ & $39.4 \%$ & 33 & 3.94 & 1.059 & \\
\hline
\end{tabular}


Table 2

The students' preference of teachers' language use for vocabulary explanation.

\begin{tabular}{lllll}
\hline & $\mathrm{N}$ & English-only & Chinese-only & Both English and Chinese \\
\hline English-only group & 35 & $16(45.7 \%)$ & 0 & $19(54.3 \%)$ \\
Chinese-only group & 33 & $12(36.4 \%)$ & 0 & $21(63.6 \%)$ \\
Control group & 34 & $16(47.1 \%)$ & 0 & $18(52.9 \%)$ \\
Total & 102 & $44(43.1 \%)$ & 0 & $58(56.9 \%)$ \\
\hline
\end{tabular}

However, the finding is important because it responds to arguments suggesting that allowing some use of L1 could potentially lead to students demanding more L1 use thus detracting from students' exposure to the target language. Such concerns seem not to be grounded in empirical evidence. In fact, it seems that students' preferences for L2 are specific and pedagogically grounded rather than seeking simply to ease work burdens.

The above findings are reflective of Macaro \& Lee (2013). In that study, a majority of the Korean students preferred their teacher to use both English and Korean in teaching English vocabulary and also preferred English-only instruction if these English explanations were understandable. While Macaro \& Lee (2013) asked their participants to respond to given statements in the questionnaire with regard to teachers' language use, the current study offered students three options of teachers' language choices in the questionnaire, forcing them to choose their preferred language choice by teachers for lexical explanations. However, Author 1 et al. (2015) reported that all three participant teachers employed the three options for lexical explanations, suggesting an incongruity between teacher practice and student preference, where the teachers were more likely than the students to tend towards L1 exclusivity. One teacher in Author 1 et al. (2015) devoted only one quarter of total explanation time to L1-only explanations, while two other teachers actually offered L1-only explanations over half of the total explanation time.

\subsection{Chinese translations}

Better comprehension: Differentiated instruction. The teacher's use of Chinese for lexical explanations was seen to be a means of adapting to students' English proficiency level. Some students evaluated themselves as being of low English proficiency, and felt that Chinese could relieve their anxiety towards an English-only environment. For example, a student from the Chinese-only group stated, "the teacher's lexical explanations is suitable to my English proficiency level, I feel relieved and comfortable" (CS $\left.{ }^{4} 24\right)$. This anxiety also echoes Guo's findings (Guo, 2007) and brings to mind the need to maintain a low affective filter in order to facilitate language learning (Daly, 1991; Horwitz, Horwitz, \& Cope, 1986). The finding is also consistent with Macaro \& Lee (2013), who found proficiency level to be a deciding factor of attitudinal differences among Korean students towards their teachers' L1 use.

Among the English-only students the reverse issue was evident, with some reporting difficulties with English-only lexical explanations, especially for low proficiency level students. Among these students English-only lexical explanations seemed to hinder their comprehension. For example, "some English explanations are very unclear, especially with difficult words such as adjective and adverbs" (ES11). The data suggests that students believe the English explanations to be too difficult and even a barrier to fully understanding the word. Proficiency was again highlighted as an influencing variable: "if the general English proficiency level is low, English explanation might cause problem of comprehension" (ES12).

Some English-only students outlined some of the specific advantages they perceived in the use of L1,"when an English explanation is hard or unclear to understand, Chinese should be used as complement, such as some nouns which are rarely used" (ES7); "Chinese meanings are quicker for understanding" (ES8); "Chinese is good with difficult words, as it helps with comprehension" (ES6). These benefits of Chinese translations listed by the English-only students echo the advantages reported by Chinese-only group. Chinese translations were seen to relieve the high cognitive load imposed by English-only explanations, in particular for low proficiency level students (Macaro, 2001). It seems that the use of L1 lexical explanations was perceived by low-level students as relieving anxiety and might also lead to better vocabulary learning outcome. Although the parent study (Author 1 et al., 2012) claimed no confirmation of the significant influence of general proficiency on vocabulary learning, for the lowest proficiency level students, those Chinese-only students who received the teacher's use of Chinese in lexical explanations recalled 24 more words than those English-only students who received English lexical explanations. Further, there were also students from the English-only group who highlighted the need for the complexity of the explanation to be appropriately pitched to the level of the student. For example, "it is better to use simple English to explain English new words" (ES6). These students stressed the importance of using simple English explanations, due to their own experience of difficulty in understanding some of the teacher's English explanations during the intervention, which revealed that the teacher's English explanations were accessible to students, but on condition that the teacher'sL2 is adapted to the level and needs of the students. In other words, if students' proficiency level allows them to understand the teacher's English explanations, these explanations are deemed as help-

\footnotetext{
${ }^{4}$ CS: Chinese-only student; ES: English-only student; CONS: control group student.
} 
ful and as bringing the advantages outlined above. For example, "the teacher used easy and simple English to explain the target words, which help me understand better" (ES8). This echoes the findings of Macaro \& Lee (2013) that English-only instruction for lexical explanation was preferred by Korean students only if English explanations were understandable. The findings point to a possible consideration in outlining what constitutes 'principled use of the L1', namely, that teachers adapt their language use according to the needs of their learners, perhaps providing more than one lexical explanation such that the explanations cater for the increasingly common mixed ability class. In order to facilitate this, it is essential to equip teachers with effective strategies for explaining difficult lexical items in the L2.

The observation of teachers' lexical explanations in Author 1 et al. (2015) revealed a wide range of strategies for explaining different lexical items, but the effectiveness of these strategies is still a concern, especially for a wide range of students. A further interpretation of the data also suggests the possibility that students might be ill-equipped to deal with L2 lexical explanations, suggesting the need for a two pronged approach where teaching is tailored to students' needs and students are equipped with the tools to access the input. Indeed, this would echo the findings reported in Macaro (2014b), where L2 learners were only able to use cognates to guess the meaning of unfamiliar words and found their teachers' L2 lexical explanations very difficult. The mismatch between teachers' English lexical explanations and students' proficiency levels should, therefore, be a major concern for teachers' decision-making in offering lexical explanations in English.

Better comprehension: L1-L2 correspondence. In the absence of teachers' use of L1, English-only students attempted to use Chinese as a mediator in order to understand the given English explanations, attempting to translate the English explanations into Chinese. For example, as expressed by Student 11, "it is hard for me to find Chinese equivalents for these English explanations, the English meanings are understood generally but not exactly" (ES11); "if an English explanation is used, I will translate it into Chinese to understand its meaning" (ES8). These comments are reflective of the importance of mental translation (Prince, 1996). Literature in this area provides evidence to support the view that the two languages are inter-woven in the L2 user's mind in vocabulary (Beauvillain \& Grainger, 1987). In the learner's mind the L2 meanings do not exist in isolation from those of the L1. On the one hand, it might be argued that this is an indication of incomplete proficiency and as such the use of the L1 in this way is viewed as a scaffolding strategy. In this sense it could be argued that, for those words that are unfamiliar to students, the L1performs a mediating role until a firm and direct link could be established between the L2 word and its concept (Jiang, 2000; Wesche \& Paribakht, 1996). On the other hand, models of compound bilingualism suggesting the two languages are simultaneously 'online' provide an alternative interpretation and offer theoretical support for pedagogical practices that reflect these processes, thus aligning external classroom learning with internal cognitive processes (Kroll \& Stewart, 1994). Such views support a view of the L1 as an unavoidable and even desirable mediator for the L2, with students employing the L1 in order to facilitate a direct link between new L2words and their concept (Jiang, 2000). More broadly such arguments relate to proposals that a connection between L1 and L2 should be seen as an indisputable fact of life and something teachers should work with rather than against (Cook, 2001; Stern, 1992).

Chinese explanations were cited by about a third of the Chinese-only students as enhancing the direct L1-L2 link for concrete words in particular. Student 18, for example, stated that, "Chinese explanation helps understand easier those English words used in daily life" (CS18). The student went on to explain that by words used in daily life she was referring to words such as vegetables, fruits, furniture and so on, arguably concrete words. For such vocabulary, L1 equivalents were regarded by some students as more helpful in comprehension of the L2 concept. Paivio (1991) stated that concrete words possess stronger image-provoking properties of L2 concepts, activating more easily the visual system in cognitive processing. Offering L1 translation enables a more direct link between the two language modes (Jiang, 2000; Van Hell and De Groot, 1998).

Better memorization. In addition to assisting with comprehension, Chinese translations also seemed to facilitate students' deployment of memorization strategies. About 1/4 of the students from Chinese-only group reported that "Chinese explanations are easy to memorize, and it takes longer to memorize English explanations" (CS13), and "Chinese meanings make new words more concrete and specific, so help with effective memorization of new words" (CS14). These comments from the interviewees indicate the importance of Chinese in assistance with memorization of new words. Students from the English-only group also reported that "Chinese meanings are quicker for understanding and easier for memorization" (ES8). Arguably, having to memorize English explanations as well as the new English lexical item, creates a greater cognitive burden of the students. Additionally, however, it is not difficult to suppose that, the Chinese explanations enable an exact and direct link between the Chinese meanings and the concepts of target English items, facilitating understanding and in turn making memorization processes easier. This idea was supported by the findings of the parent study (Author 1 et al., 2012), which indicated that the Chinese-only group recalled significantly more target words than the English-only group in the immediate post-tests. It also echoes findings by Macaro \& Lee (2013:734), suggesting that L1 equivalents allowed students to "immediately understand what the word meant, but also link it to other information ... more likely to ensure its acquisition." Furthermore, the empirical findings of Zhao \& Macaro (2014) lend credence to the idea that providing L1 translation of lexical items allows students to perform better than providing an L2 only explanation, both at post and delayed vocabulary tests. 


\subsection{English lexical explanations}

Linguistic immersion. ${ }^{5} 80 \%$ of students from the English-only group believed a key advantage of English explanations to be their role in creating what they referred to as an English 'immersion' environment, which they felt was beneficial, particularly for their listening comprehension skills. It is important to remember that these participants were English-major students at the university, among whom prevails a strong belief in using the target language (Kong \& Zhang, 2005). Such a belief is in one sense supported by literature arguing for the need for sufficient comprehensible input as a precondition for language acquisition (Krashen, 1988). Students in the Chinese-only group also expressed this belief when commenting on the drawbacks of Chinese translations, claiming that, "as an English-major, English-only environment is crucial to help create a good atmosphere" (CS16). Furthermore, when commenting on their preferences on teachers' language choice in lexical explanations, half of them expressed the same worries as the English-only group, that the use of Chinese-only explanations might potentially undermine their exposure to the target language environment. In addition, half of the students from the control group who did not receive any lexical treatment also reported the same concern.

Indeed, while certain benefits of principled L1 use have been acknowledged, the literature does support the significance of the input environment. Immersed in a one-way English learning environment, learners can achieve as well as and at times better than their non-immersion peers (Genesee, 2008) and this can be seen to apply regardless of socio-economic and ethnic backgrounds or even whether the second language is alphabet-based or character-based (Lindholm-Leary, 2001; Slaughter, 1997). This concern from the English-major students on the target language environment, regardless of the different linguistic medium of lexical explanation they received, echoes the underlying concerns of many teachers in China, striving to create a linguistic immersion environment in their EFL classrooms (Guo, 2007). Similarly, the adult Korean learners in the study of Macaro \& Lee (2013) were also acutely aware of the importance of being exposed to English environment. Although not clearly outlined in governmental and institutional linguistic policies, it is a widely accepted doctrine in China that an exclusive English environment is vital for English learners to acquire the language.

Improved accuracy of comprehension: Half of the students from the English-only group indicated that English explanations could help them accurately understand the meanings of target words. For example, "English explanations help with accurate understanding the meanings of English words" (ES3). That is, English explanations were considered by this group, to be more closely and accurately related to the meanings of target words, seemingly implying that certain elements of the meaning might be lost in the process of translation (Butzkamm, 2003). As reported by students, "sometimes the English explanations would help with better understanding the words with similar meanings than the Chinese explanations" (ES4). English explanations could help distinguish synonyms while the Chinese translations for some English synonyms might be the same, particularly where distinctions in the L2 do not exist in the L1. ${ }^{6}$ This loss of meaning in translation has been found to occur particularly with abstract rather than concrete words because of the lesser likelihood of shared conceptual features (Kroll \& De Groot, 1997:187). Abstract words across languages are more likely to have roughly similar equivalents, rather than close translations. The more abstract the L2 words are, the more distant the meaning match is likely to be between the two languages (Kroll \& De Groot, 1997). In such cases, therefore, English explanations, although lengthy, provide closer and more accurate meanings. As reported, "the teacher gave us the English explanations, it's long but it is very vivid, I could understand the meanings" (ES4).

\subsection{A bilingual approach of lexical explanations}

Over half of the students from the three groups held a preference for a bilingual approach in teacher's lexical explanation; that is, the teacher using both English and Chinese as the linguistic medium for explaining vocabulary. Macaro \& Lee (2013) recommended a bilingual approach to lexical explanations, but did not go further in operationalizing what such explanations might look like. The qualitative analysis of students' preferred perceptions presented in this study goes some way to begin addressing this point.

In general, students believe that when they encountered difficulties in understanding English explanations or when some words were too difficult to explain in English, Chinese could be used. For the English-only group who received English-only lexical explanations, five out of the nine interviewees argued that English should be used as the language for lexical explanations, but "when students have difficulties in understanding English explanation, Chinese should be used to help with understanding. Otherwise, the meanings of words are still not understood" (ES12). This seems rather a deficit view of the L1, recommending it for use only when the L2 cannot suffice. Such a view can be seen as echoing the 'maximal position' that some teachers might hold and outlined by Macaro (2001), whereby the use of L1 is permitted as a concession to the fact that perfect teaching conditions do not exist and teachers must therefore resort to L1 use.

\footnotetext{
${ }^{5}$ It should be noted that an immersion environment involves far more than simply explaining new vocabulary in L2. The linguistic immersion environment refers to the exclusively English language use by the teacher of this study within the EFL classroom.

${ }^{6}$ For example, the Chinese translation for empty and blank is the same but these two words actually refer to different things.
} 
Similar views were reported by students from the Chinese-only group. Although $63.6 \%$ of the students reported a preference for a bilingual approach in the questionnaire, all fourteen interviewees agreed that there was value in both languages for lexical explanations, but that English should be the main language, with Chinese used to aid understanding of difficult words. Indeed half of the control group participants agreed with this, expressing views such as "it's better to use English more of the time, but for complex ones Chinese would help" (CONS25) and "some proper words can be explained in English, and those difficult to understand can then be explained in Chinese" (CONS31). It is notable that the first recourse when encountering difficult lexical explanations is to resort to an L1 translation rather than deploying other strategies, an issue discussed previously.

To summarize, the contextualized perceptions and preferences of students towards teachers' Chinese-only and English-only lexical explanations in an EFL university context with regard to the learning of lexis brings valuable insights into understanding teachers' classroom language use for lexical explanations from the perspective of students. Students were generally satisfied with their teachers' language use for lexical explanations, but they all preferred teachers' lexical explanations to be delivered either in L2-only or through a mixture of L1 and L2. Students have perceived teachers' use of English and use of Chinese for lexical explanations as facilitative to the comprehension and memorization of English lexis from different perspectives.

\section{Conclusion and implications}

Questionnaire data indicated that overall students are satisfied with their teachers' L1 use and find it helpful for their understanding and learning. Since this was the case in both Chinese-only and English-only groups, it would seem to imply that the lexical explanations were well-suited to the level of the students.

An analysis of students' qualitative responses reveals more valuable insights into the distinctions students made between use of English and use of Chinese for lexical explanations. Chinese was seen to assist with better comprehension and to be a useful means through which to tailor instruction to students' proficiency levels. Additionally, it was believed to offer more precise understanding through a direct L1-L2 correspondence, thus facilitating memorization. On the other hand English was believed to be particularly helpful in assisting students to understand the fine distinction between similar words, particularly where these distinctions may not be present in Chinese.

A key problem of English lexical explanation was seen to be the mismatch between the complexity of teacher's lexical explanations and students' proficiency levels, which was reported to impede students' comprehension and memorization of target English words. This suggests a need for teachers to be equipped with a range of teaching strategies to be able to convey complex lexical items in simple English, suited to the proficiency level of their students. In a mixed ability setting this requires particular expertise on the part of the teacher to be able to provide a range of explanations catering for the range of needs.

In terms of preferences towards teachers' language use in lexical explanations, all the participants, including the control group, expressed a preference for lexical explanations to be delivered either in L2-only or through a mixture of L1 and L2. Such a preference is incongruent with the teachers' actual lexical explanation behavior observed by Author 1 et al. (2015), whereby teachers employed the three options for lexical explanations. Students' expressed need for L1 explanations was in the context of L2 explanations also, rather than in an exclusive L1 context, reflecting their concerns that exclusive L1 lexical explanations might potentially undermine their exposure to the L2.

The views expressed by the students, by implication demand teachers to make critical pedagogical decisions on what type of lexical explanation requires which language and when. In such a view, teachers function as both dictionary and dictionary designer (Author 1 et al., 2009) as they have an array of choices at their disposal, for example L1 or L2 paraphrase, definition, synonym or contextualization. Student data examined in this study would suggest that English explanations should be given first; the subsequent use of Chinese explanations would depend on students' comprehension of the English explanation. If students could understand the English explanation of unknown lexical items, then arguably the Chinese explanations would not be necessary; but if there is difficulty in students' comprehension of English explanations, teachers could use Chinese to assist comprehension. That is, an English explanation should be given first followed by a Chinese translation only when incomprehensibility arises. A series of factors might impact the above decision-making process of teachers. One factor is students' proficiency level, which emerged as the primary concern of many students from different groups in this study. For those with lower proficiency levels, the comprehensibility of teachers' English explanations bears topmost priority. Teachers then could offer Chinese input to assist comprehension. Another factor could be the level of concreteness of L2 words. Based on the findings, concrete words are more likely to require an L1 translation and abstract words might more easily be explained in L2, particularly where no clear L1 parallel exists. These proposals require teachers to be highly skilled and adaptable in their ability to adapt their language use according to students' proficiency and the nature of the vocabulary taught. This requires considerable planning and, as such, they need to be trained with a wide range of linguistic and pedagogical strategies on how to make decisions about the best form of explanation and on how to deliver level-appropriate lexical explanations. Students should also be trained with an array of learning strategies to cope with the different demands of language use in class, in order to enable them to move beyond a translation-only approach that does not equip them to deal with the range of vocabulary they are exposed to.

Future research could extend understandings of students' perceptions of teachers' lexical explanations to a wider and more diverse population of students, examining whether perceptions vary, for instance according to subject major or type of lexis. It is possible, for example, that technical vocabulary requires different types of lexical explanations. Such research would be particularly significant and interesting in contexts where content-based instruction is implemented. Also, a larger population of students 
could allow further in-depth examination into the significant effect of learner proficiency levels on their preference of L1 and L2 lexical explanations. Additionally, further research might usefully examine the effectiveness of the proposed bilingual approach principles for teaching different word types. Finally, research looking at the specific nature of lexical explanations, in other words how teachers actually formulate their explanations, and their impact on students' learning would make a useful and insightful contribution to the field, particularly if coupled with an investigation of teachers' accompanying cognitions.

\section{Acknowledgement}

We feel very grateful for all the comments raised by the reviewers. We also would like to express our gratitude for the participant students who contributed greatly to the study. This study was supported by the Fundamental Research Funds for the Central Universities, and the Research Funds of Renmin University of China. 


\section{Appendix 1. Questionnaire}

Student number

Gender

Which province and city are you from?

Please rate your current general proficiency in English by choosing one that matches you best in the following scale from 1 (poor) to 5 (very good)

$\begin{array}{lllllll}1 & 2 & 3 & 4 & 5 & 6 & 7\end{array}$

Please rate your current proficiency in English Listening by choosing one that matches you best in the following scale from 1 (poor) to 5 (very good)

$\begin{array}{lllllll}1 & 2 & 3 & 4 & 5 & 6 & 7\end{array}$

Please rate your current proficiency in English vocabulary by choosing one that matches you best in the following scale from 1 (poor) to 5 (very good)

$\begin{array}{lllllll}1 & 2 & 3 & 4 & 5 & 6 & 7\end{array}$

How many sessions of this experimental training session have you attended?

$\begin{array}{llllll}1 & 2 & 3 & 4 & 5 & 6\end{array}$

How helpful are the teachers' explanations of the background information on the listening passages in terms of understanding these listening passages? Please choose the one that matches best in the following scale from 1 (not at all helpful) to 5 (very helpful)

$\begin{array}{lllll}1 & 2 & 3 & 4 & 5\end{array}$

Please state briefly the reason for your answer

How helpful are the teachers' explanations of the unknown words in terms of learning the vocabulary from the listening passages? Please choose the one that matches best in the following scale from 1 (not at all helpful) to 5 (very helpful)

$\begin{array}{lllll}1 & 2 & 3 & 4 & 5\end{array}$

Please state briefly the reason for your answer

Are you satisfied with the teachers' choice (English or Chinese) of language use in explaining the unknown words? Please choose the one that matches best in the following scale from 1 (not satisfied at all) to 5 (very satisfied)

$\begin{array}{lllll}1 & 2 & 3 & 4 & 5\end{array}$

Please state briefly the reason for your answer

Which type of language choice do you think the teacher in this training session should use to explain the unknown words? Please choose the one that matches best among the following choices.
a. English
B. Chinese
C. English and Chinese

\section{Appendix 2. Interview questions:}

English-only Group.

1. How do you generally study English new words? What help you grasp these words? What methods have you used?

2. How do you learn the new words in the listening passages during this training session?

3. How do you feel when the teacher in the training session explain the new words in English?

4. Do you think the teacher's explanation helps? 
5. If you could choose, which type of language do you think the teacher should use, Chinese or English, to explain the new words so as to help you comprehend and memorize them? Why?

Chinese-only Group.

1. How do you generally study English new words? What help you grasp these words? What methods have you used?

2. How do you learn the new words in the listening passages during this training session?

3. How do you feel when the teacher in the training session explain the new words in Chinese?

4. Do you think the teacher's explanation helps?

5. If you could choose, which type of language do you think the teacher should use, Chinese or English, to explain the new words so as to help you comprehend and memorize them? Why?

\section{Control Group.}

1. How do you generally study English new words? What help you grasp these words? What methods have you used?

2. Could you describe when you heard the three listening passages what were you thinking?

3. What were you thinking when a new word comes up in the listening passage?

4. Do you think the teacher's introduction on the TEM-4 exam benefit you? How?

5. If you could choose, which type of language do you think the teacher should use, Chinese or English, to explain the new words so as to help you comprehend and memorize them? Why?

\section{Uncited references}

Liao, 2006; Macaro, 2014a; Polio and Duff, 1994; Tanaka and Ellis, 2003.

\section{References}

Anton, M., DiCamilla, F., 1998. Socio-cognitive functions of L1 collaborative interaction in the L2 classroom. The Canadian Modern Language Review $54,314-342$

Beauvillain, C., Grainger, J., 1987. Accessing interlexical homographs: Some limitations of language-selective access. Journal of Memory and Language 26 (6), $658-672$.

Butzkamm, W., 2003. We only learn language once. The role of the mother tongue in FL classrooms: Death of a dogma. Language Learning Journal 28, 29-39.

Cook, V., 2001. Using the first language in the classroom. The Canadian Modern Language Review 57, 402-423.

Daly, J., 1991. Understanding Communication Apprehension: An introduction for language educators. In: Horwitz, E., Young, D.J. (Eds.), Language Anxiety: From theory and research to classroom implications. Prentice Hall, Englewood Cliffs, NJ, pp. 3-13.

Duff, P.A., Polio, C.G., 1990. How much foreign language is there in the foreign language classroom?. The Modern Language Journal 74, $154-166$.

Gao, J., Dai, W., 2007. An empirical study on codeswitching in language teaching. Foreign Language Teaching 28 (1), 51-55.

Genesee, F., 2008. Dual language in the global village. In: Fortune, T.W., Tedick, D.J. (Eds.), Pathways to multilingualism: Evolving perspectives on immersion education. Multilingual Matters, Ltd, Clevedon, England, pp. 22-45.

Guo, T., 2007. A case study of teachers' code-switching behaviors in mainland China's university EFL classrooms and students' reactions to the codeswitching. University of Oxford. Unpublished DPhil Thesis.

Horwitz, E.K., Horwitz, M.B., Cope, J.A., 1986. Foreign language classroom anxiety. Modern Language Journal 70, 125-132.

Jiang, N., 2000. Lexical representation and development in a second language. Applied Linguistics 21 (1), 47-77.

Kong, H., Zhang, P., 2005. An investigation on teacher talk in an Extensive Reading classroom for English majors. Education and Modernization 76 (3), 38-43.

Krashen, S.D., 1985. The input Hypothesis: Issues and implications. Longman, New York.

Kroll, J., De Groot, A., 1997. Lexical and conceptual memory in the bilingual: Mapping form to meaning in two languages. In: de Groot, A.J., Kroll, J. (Eds.), Tutorials in Bilingualism: Psycholinguistic perspectives. Lawrence Erlbaum Associates Publishers, Mahwah, New Jersey.

Kroll, J., Stewart, E., 1994. Category interference in translation and picture naming: Evidence for asymmetric connections between bilingual memory representations. Journal of Memory and Language 33, 149-174.

Lee, J.H., Macaro, E., 2013. Investigating age in the use of L1 or english-only instruction: Vocabulary acquisition by Korean EFL learners. The Modern Language Journal 97 (4), 887-901.

Levine, G.S., 2003. Student and instructor beliefs and attitudes about target language use, first language use, and anxiety: Report of a questionnaire study. The Modern Language Journal 87, 343-364.

Liao, P., 2006. EFL Learners' beliefs about and strategy use of translation in English learning. RELC Journal 37 (2), 191-215.

Lindholm-Leary, K., 2001. Dual language education. Multilingual Matters Ltd, Clevedon, England.

Macaro, E., 1997. Target language, collaborative learning and autonomy. Multilingual Matters LTD.

Macaro, E., 2001. Analysing student teachers' code-switching in foreign language classrooms: Theories and decision making. The Modern Language Journal $85,531-548$.

Macaro, E., 2014a. Overview: Where should we be going with classroom codeswitching research?. In: Barnard, R., McLellan, J. (Eds.), Codeswitching in university English-medium classes: Asian perspectives. Multilingual Matters, Bristol, England, pp. 10-23.

Macaro, E., 2014b. Students' strategies in response to teachers' second language explanations of lexical items. Language Learning Journal 1-16.

Macaro, E., 2015. The teacher's codeswitching and the learner's developmental response: Pursing a research agenda [Seminar to Faculty of Education, HKU]. University of Hong Kong. 15th June.

Macaro, E., Lee, J.H., 2013. Teacher language background, codeswitching, and english-only instruction: Does age make a difference to learners' attitudes?. TESOL Quarterly 47 (4), 717-742.

Miles, M., Huberman, A., 1994. Qualitative Data Analysis: An Expanded Sourcebook. Sage Publications, Thousands Oaks. 
Paivio, A., 1991. Dual coding Theory: Retrospect and current status. Canadian Journal of Psychology 45 (3), $255-287$.

Polio, C.G., Duff, P.A., 1994. Teachers' language use in university foreign language classrooms: A qualitative analysis of english and target language alternation. The Modern Language Journal 78, 313-326.

Prince, P., 1996. Second language vocabulary learning: The role of context versus translations as a function of proficiency. The Modern Language Journal $80,478-493$.

Slaughter, H., 1997. Indigenous language immersion in Hawai'i: A case study of Kula Kaiapuni Hawai'i, and effort to save the indigenous language Hawai'i. In: Johnson, R.K., Swain, M. (Eds.), Immersion education: International perspectives. Cambridge University Press, Cambridge, pp. $105-129$.

Stern, H.H., 1992. Issues and options in language teaching. Oxford University Press, Oxford.

Tanaka, K., Ellis, R., 2003. Study-abroad, language proficiency, and learner beliefs about language learning. Japan Association for Language Teaching 25 (1), 63-85.

Van Hell, J., De Groot, A., 1998. Disentangling context availability and concreteness in lexical decision and word translation. The Quarterly Journal of Experiment Psychology 51 (1), 41-63.

Wesche, M., Paribakht, T.S., 1996. Assessing second language vocabulary knowledge. Canadian Modern Language Review 53 (1), $13-40$.

Zhao, T., Macaro, E., 2014. What works better for the learning of concrete and abstract words: Teachers' L1 use or L2-only explanations?. International Journal of Applied Linguistics http://dx.doi.org/10.1111/ijal.12080.

Zhong, X., 2007. Using english/Chinese code-switching as a strategy for the english intensive-reading teaching. Journal of Chongqing Institute of Technology (Natural Science Edition) 21 (7), 162-164. 